\title{
MARTÍN SANCHO (SIGLOS XIV-XVI) UN DESPOBLADO BAJOMEDIEVAL EN LA TIERRA DE MEDELLÍ́N*
}

\section{JULIÁN CLEMENTE RAMOS}

Universidad de Extremadura

RESUMEN: Martín Sancho se documenta por primera vez en 1374. En la segunda mitad del siglo XIV, lo que quizás ha sido inicialmente una apropiación individual ha dado origen a un poblamiento concentrado. Su población nunca debió superar los veinticinco vecinos. Esta aldea contaba con su propio término y su concejo, que contaba como único oficial con un alcalde. Sus vecinos eran feligreses de la iglesia de San Martín de Medellín Hacia mediados del XV, Martín Sancho presentaba un cierto declive demográfico, no superando su población los quince-veinte vecinos, de los cuales sólo una parte residian de forma permanente en el lugar. Esta situación ha debido aumentar el peso de la ganadería, ante la abundancia relativa de pastizales, y quizás también la acumulación de propiedades por parte de miembros cualificados de la villa. En este contexto, la violencia del gobernador de la tierra y futuro señor Rodrigo Portocarrero, que adehesa el término, produce una rápida despoblación. En el primer cuarto del siglo XVI, Medellín consigue finalmente, a través de un proceso judicial, integrarlo en sus ejidos.

Palabras clave: Historia rural. Despoblados. Baja Edad Media. Extremadura.

ABSTRACT: The Extremaduran village of Martin Sancho is documented for the first time in 1374. In the second half of the fourteenth century, something that may initially bave been an individual appropriation has given rise to a concentrated settlement. Its population seems never to have been more than twenty-five. This village had its own district and council, whose only official was the mayor. Its inbabitants were parishioners of the church of San Martin de Medellin. Around the middle of the

* Trabajo elaborado dentro del proyecto «Fuentes documentales para la historia de Extremadura» (ref. 2PR02A036), financiado por la Consejería de Educación, Ciencia y Tecnología de la Junta de Extremadura. 
fifteenth century, Martin Sancho experienced a certain demographic decline, its population no more than 15-20, of whom only a part resided there permanently. This situation appears to have increased the importance of cattle raising, in the context of the relative abundance of grasses and perhaps also the accumulation of properties on the part of qualified members of the village. The violence of the governor of the tierra and future landlord Rodrigo Portocarrero, who appropriates the district, produces rapid depopulation. In the first quarter of the sixteenth century, Medellin manages through a judicial process to integrate it in its public land.

KEY WORDS: Rural History. Lost Villages. Later Middle Ages. Extremadura.

El fenómeno de los despoblados, wüstungen o lost villages tiene una importancia considerable en la baja Edad Media ${ }^{1}$, fase que comienza con una clara recesión demográfica que se prolonga, en función de las distintas regiones, hasta avanzado el siglo XV. La desaparición de aldeas, salvo excepciones muy contadas de escasa entidad demográfica y a veces de ubicación no demasiado favorable $^{2}$, no puede explicarse meramente como consecuencia de una coyuntura demográfica, sino que debe entenderse dentro de un proceso de transformación y consolidación del poblamiento. No debemos olvidar, por otro lado, que toda nueva ocupación del espacio está lleno de tanteos y múltiples fracasos ${ }^{3}$. Los despoblados son parte integrante del mundo rural a lo largo de toda la Edad

1 ABEL, W.: Crisis agraires en Europe (XIII-XX siècles), ParÍs, 1973, págs. 115-116; HeERS, J.: Occidente durante los siglos XIV y XV. Aspectos económicos y sociales, Barcelona, 1984, págs. 345-6; Berthe, M.: Famines et epidémies dans les campagnes navarroises à la fin du Moyen Age, Paris, 1984, vol. 1, págs. 490-5; RodrígueZ GaLdo, M.X.: Señores y campesinos en Galicia. Siglos XIV-XVI, Santiago, 1976, págs. 55-6; GARCía DE CORTÁZAR, J. A.: La sociedad rural en la España medieval, Madrid, 1988, págs. 189-190; REGLERO DE LA FuENTE, C.: «Los despoblados bajomedievales en los montes Torozos: jerarquización del poblamiento y coyuntura económica», Edad Media. Revista de Historia, 1 (1998), pág. 186; E. Portela Silva, La región del obispado de Tuy en los siglos XII a XV. Una sociedad en la expansión y en la crisis, Santiago, 1976, págs. 281-3.

2 ABEL, W.: Crisis agraires en Europe, pág. 118; Id., «Désertions rurales: bilan de la recherche allemande», Villages desertes et histoire economique (XI-XVIII siècles), Paris, 1965, págs. 523-4; M. W. BERESFORD, «Villages désertés: bilan de la recherche anglaise», Ibíd., págs. 552-3; la despoblación ha afectado poco a la estructura parroquial: PESEZ, J. M. y LE ROY LADURIE, E.: «Le cas français: vue d'ensemble», Ibíd., pág. 190; Díaz De Durana, J. R.: Alava en la baja Edad Media. Crisis, recuperación y transformaciones socioeconómicas (c. 1250-1525), Vitoria, 1986, págs. 124-5; VACA LORENZO, A.: «La estructura socioeconómica de la Tierra de Campos a mediados del siglo XIV», Public. Instituc. Tello Téllez de Meneses, no 39 (1977), págs. 385-9; Reglero de LA Fuente, C.: «Los despoblados bajomedievales en los montes Torozos», pág. 199.

3 Martínez Sopena, P.: La Tierra de Campos occidental. Poblamiento, poder y comunidad del siglo $X$ al XIII, Valladolid, 1985, págs. 181-9; CABrillanA, N.: «Salamanca en el siglo XV: nobles y campesinos», Cuadernos de Historia. Anexos de la Revista Hispania, 3 (1969), pág. 284; Clemente RAMOS, J. y DE LA MONTAÑA, CONCHIÑA, J. L.: «La Extremadura cristiana medieval. (1142-1230). Ocupación del espacio y transformaciones socioeconómicas», Historia. Instituciones. Documentos, 21 (1994), pág. 96. 
Media, pero empiezan a ser raros a partir de comienzos de la Moderna. Aunque no ha sido estudiada sistemáticamente, la documentación extremeña alude a aldeas que han terminado convirtiéndose en meras dehesas ${ }^{4}$. La ocupación inicial y la casi definitiva configuración del poblamiento en Extremadura son realidades temporalmente muy próximas debido a su tardía conquista.

Los despoblados no fueron simplemente producto de un proceso de racionalización de la explotación del territorio. A ello se une la actuación de agentes exógenos, entendiendo como tales a todos aquellos factores ajenos directamente a la población o al aprovechamietno del espacio. La violencia señorial ocupa un papel importante en algunos procesos 5 , y desde luego es el elemento fundamental en el que vamos a estudiar, aunque nunca funciona como factor único. Difícilmente consigue la despoblación de una población de cierta entidad. Más bien es una casuística que incide en situaciones avanzadas o terminales. Aparece como último factor en el sentido cronológico.

Por razones obvias, los despoblados han sido abordados a escala comarcal o regional, método que permite situar estos ejemplos dentro de su contexto general. Por otro lado, es raro que la información se acumule para un núcleo poblacional. En muchos casos, no disponemos de información escrita sino solo arqueológica. Para Martín Sancho, la situación es diferente. Los pleitos sobre este antiguo término aldeano nos suministran una información de gran interés, sobre todo para el momento inmediatamente anterior a su despoblación ${ }^{6}$. Nos permiten, también, trazar su aprovechamiento posterior hasta su definitiva incorporación, tras una primera apropiación por parte de los Portocarrero, a los ejidos de Medellín. Estamos ante un ejemplo de microhistoria: los datos nos permiten analizar un caso muy particular y ahondar en el proceso de despoblación valorando todos los factores.

4 Este fenómeno alcanza gran amplitud en la tierra de Medellín, que actualmente estamos estudiando. Todos los despoblados se transforman en dehesas. Su número se eleva a cinco en una jurisdicción donde existen once núcleos de población a finales de la Edad Media. Posiblemente, no estamos ante un caso especial. Dentro de la Submeseta Sur, la Alcarria ofrece un panorama parecido (DíAz LEÓn, A. Ma , Gómez Bravo, M. y Ma I. MARTínez MARTín, «El despoblado medieval de La Golosa [Berninches, Guadalajara]. I campaña arqueológica (1991)», Wad-Al-Hayara, 20 [1993], págs. 53-4).

5 Cabrillana, N.: «Salamanca en el siglo XV», págs. 269-270; para Soria, AsENJO, Mª: «Las tierras de baldío en el concejo de Soria a fines de la Edad Media», Anuario de Estudios Medievales, 20 (1990), págs. 409-410, considera la violencia un elemento desencadenante de la despoblación; una posición contraria mantiene Diago HeRnANDO, M.: «Los términos despoblados en las comunidades de villa y tierra del Sistema Ibérico castellano a finales de la Edad Media», Hispania, LI/2 (1991), págs. 474-6

6 A. Ch. Granada, legs. 1083, nº 7; 1371, nº 5; y, sobre todo, leg. 577, no 24 (años 1494 a 1520). 


\section{EL ORIGEN DE MARTÍN SANCHO}

Desconocemos la fecha en que Martín Sancho surge como entidad poblacional. La referencia más antigua es de 1374 y nos ofrece algunos datos de gran importancia. Se alude en ella a «el exido de Martin Sanchez, vezino de la dicha mi villa» ${ }^{7}$. Sin duda, estamos ante el término de Martín Sancho, pues se sitúa al sur de la dehesa de la Patilla y próximo al Ruecas. Esta alusión plantea algunas cuestiones. No se señala en ningún caso que haya una aldea en dicho ejido, sólo se alude a la existencia de una apropiación. En todo caso, dicha apropiación es algo reciente, pues la persona que la ha realizada es, en ese momento, un vecino de la villa. Podría retrasarse esta acción alguna década, en todo caso no sería muy anterior a mediados del XIV aceptando la hipótesis más temprana. La utilización del término ejido alude con claridad al carácter comunal del terreno. Razonablemente debemos pensar que hacia mediados del XIV, quizás un poco antes por razones que luego veremos, coincidiendo con un momento en el que aparecen síntomas de dinamismo demográfico en la tierra de Medellín y en Extremadura en su conjunto ${ }^{8}$, Martín Sanchez (o Sancho) se ha apropiado de un terreno comunal. Es posible que se haya establecido en el mismo un poblamiento de carácter disperso, acorde con el carácter individual de la propiedad.

Hasta el momento inmediatamente anterior a la despoblación, sólo disponemos de una información aislada, pero también presenta un gran interes. En 1391, Alfonso Fernández de Medellín, hijo de Juan Fernández Asturiano, lega en su testamento a Leonor Alfonso, mujer de Juan Sánchez de Medellín, «la quarta parte de dies e ocho yugadas de tierra de heredamiento en Martin Sancho, aldea de Medellín, e un solar de casas»`. Sin duda, se plantea una realidad bien distinta menos de veinte años después de la primera mención documental. El nuevo núcleo poblacional se cita como una de las aldeas de Medellín, no mencionándose su antiguo carácter de ejido. Por otro lado, si fue, como parece, objeto de una apropiación individual, en este momento se ha constituido un hábitat concentrado. La propiedad se halla distribuida entre un número indeterminado de propietarios y, sin duda, entre personas ajenas a Martín Sancho o Sánchez. Alfonso Fernández de Medellín no tiene nada que ver, o eso parece, con este personaje. En todo caso, no se alude a esta circunstancia cuando se presenta esta escritura en uno de los pleitos señalados.

En conjunto, podemos sintetizar unas líneas genéricas pero en absoluto carentes de interés. En torno a mediados del siglo XIV, quizás un poco antes, se ha realizado una apropiación sobre un espacio comunal de Medellín. Es posible

7 Cerro Herranz, Ma F.: Documentación del monasterio de. Guadalupe. Siglo XIV, Badajoz, 1987, doc. 127.

8 SÁnCHeZ Loro, D.: Historias inéditas placentinas, II, Cáceres, 1983, págs. 232-233; CLEMENTE RAMOS, J.: «El medio natural en la vertiente meridional del Tajo extremeño en la baja Edad Media», Anuario de Estudios Medievales, 30/1 (2000) , pág. 322.

9 A. Ch. Granada, leg. 1371, no 5, fol. 26v. 
que haya dado origen a un hábitat disperso y a una apropiación individual. En todo caso, décadas más tarde, Martín Sancho se ha transformado en una aldea y la propiedad debía repartirse entre un número indeterminados de vecinos o simplemente propietarios.

De todos modos, no podemos excluir que desde el primer momento, Martín Sancho haya sido una proyecto de aldea, lo que explica que Medellín no se haya opuesto a su desarrollo. En el momento de su constitución, al menos en la segunda mitad del siglo XIV, es la que se sitúa más próxima a la villa. Con la excepción de Mengabril, documentada más tardíamente, el resto de la aldeas existentes en el siglo XVI tienen una posición periférica dentro de la tierra de Medellín, lo que no deja de ser sintomático. Posiblemente fue un factor que condicionó negativamente la evolución de Martín Sáncho ${ }^{10}$.

\section{MARTÍN SANCHO A MEDIADOS DEL SIGLO XV}

La información es abundante sobre la quinta década del siglo XV, es decir en los últimos años de la existencia como aldea de Martín Sancho. Procede de interrogatorios realizados en general medio siglo más tarde o poco más. La información la consideramos muy fiable. Aunque hablando de hechos acaecidos entre cuarenta y sesenta años antes, es detallada y precisa. Cuando se puede constrastar con datos documentados, la fiabilidad cronológica es elevada. La información disponible nos permite reconstruir con bastante detalle la realidad de una pequeña comunidad campesina.

El asentamiento goza de una buena ubicación. El terreno es llano y de naturaleza sedimentaria ${ }^{11}$. Sin duda se trata de un lugar que por sus suelos profundos reúne muy buenas condiciones para la agricultura. Estas mismas condiciones, sin embargo, son aplicables a la mayor parte del término de Medellín, lo que no ha impedido el desarrollo de la actividad ganadera y la proliferación de dehesas.

Martín Sancho nunca dejó de ser una pequeña comunidad campesina, demasiado reducida para disponer de una iglesia parroquial ${ }^{12}$. La unanimidad sobre el particular es total. Hacia 1449-1450, sólo tendría entre quince y vein-

10 Reglero de la Fuente, C.: «Los despoblados bajomedievales en los montes Torozos», pág. 204; Lorenzo Pinar, F. J. y IZquierdo Misiego, J. I.: «Términos redondos y despoblamiento en Ávila al inicio de la Edad Moderna. Aproximación histórica», Studia Zamorensia (2a época), VI (2002), pág. 269.

11 Hernández Pacheco, F.: Características geográficas y geológicas de las Vegas del Guadiana, Badajoz, 1956, especialmente págs. 93-5 y 97-9.

12 A. Ch. Granada, leg. 577, $\mathrm{n}^{\circ}$ 24, fol. 23r: «hera avido e tenido por arrabal de Medellín e los vesinos de Martin Sancho heran feligreses e parrochanos de la yglesia de señor San Martin de la dicha villa... e pagavan sus diezmos de lo que labravan e cogian». 
te vecinos ${ }^{13}$. Ningún testigo da una cantidad superior. Algunos hablan sólo de quince. El testigo dieciseis de uno de los interrogatorios conservados señala que «seyendo este testigo mochacho pequeño morando con su padre dormio una noche en el logar de Martin Sancho, estando poblado de hasta quinze vezinos» ${ }^{14}$. El trece estima una población de «hasta diez o doze vezinos» ${ }^{15}$.

Martín Sancho era, por tanto, una núcleo de muy escasa entidad demográfica. Sin embargo, casi un siglo despues, Villar de Rena sólo contaba con 53 vecinos, La Mancha con 28 y Cristina con $63^{16}$, pese a que la población debió cuando menos duplicarse a lo largo del siglo $\mathrm{XV}^{17}$.

No es, por tanto, la escasa entidad demográfica razón suficiente para explicar el éxito de Rodrigo Portocarrero, señor de Medellín, en la despoblación final de Martín Sancho. No debemos olvidar que frente a esta núcleo, próximo a Medellín y al Guadiana, la ubicación de las tres aldeas citadas es claramente periférica. Un factor fundamental es que estamos ante una aldea en franca crisis, en clara regresión demográfica. Hay informaciones de naturaleza diferente que inciden en la misma dirección. Martín Sancho había llegado a albergar una población superior, aunque no mucho más elevada. Dos testimonios diferentes avalan esta opinión. Francisco Montero, vecino de Don Benito, de 38 años, señala que «a visto el sitio donde el dicho lugar estava poblado, e que aun oy dia le ha visto e que bien mirado y tanteado podria aver en las casas que eran veynte e çinco sitios poco mas o menos» ${ }^{18}$. En un memorial elaborado no después de 1517 por el alcalde Juan Guisado, el bachiller Francisco Bernal y Francisco Cervigón, se afirma que «el sytio de dicho logar hera de veynte o veynte e çinco casas con sus corrales» ${ }^{19}$. Por tanto, y a través de inspecciones oculares realizadas en el primer cuarto del siglo XVI, permanecían los solares corres-

13 Gonzalo Martos, vecino de San Pedro, señala que «le conosçia poblado este tienpo de espaçio de ocho o diez años [1440-1450 aprox.]... e en el lugar bivian en el lugar quinze o veynte vezinos poco mas o menos» (A. Ch. Granada, leg. 577, $\mathrm{n}^{\circ} 24$, fols. 49r; esta misma cantidad es avalada por otros muchos testigos, Id., fols. 49r-v).

14 A. Ch. Granada, leg. 577, $\mathrm{n}^{\circ} 24$, fol. 51r (no conocemos la fecha exacta de este interrogatorio, que no debió ser muy posterior a 1500). La misma valoración realizan los testigos 12 y 15 de este mismo interrogatorio: Id., fols. $51 \mathrm{v}$ y $52 \mathrm{r}$.

15 A. Ch. Granada, leg. 577, no 24, fol. 51v.

16 Cabrera Muñoz, E. y Lora Serrano, G.: «Datos sobre la población y la configuración jurisdiccional de Extremadura en el tránsito de la Edad Media a la Moderna», 'Ifigea, I (1984), pág. 73.

17 No tenemos datos directos para la tierra de Medellín en su conjunto, aunque si para Valdetorres que sin embargo constituye un ejemplo muy particular, pues en 1409 era una dehesa con dos o tres casas y en 1531 contaba con 183 vecinos (Clemente RAMOS, J.: «Valdetorres, de dehesa a aldea [1409-1510]. Poblamiento, conflicto y poder en la tierra de Medellín», Studia Historica. Historia Medieval, 20-21 [2002-3], pág. 50; CABRERA MuÑOZ, E. y LORA SERRANO, G.: «Ibíd.», pág. 73). Para otras comarcas extremeñas, cf. BORRERO FERNÁNDEZ, M.: «El concejo de Fregenal: población y economía en el siglo XV», Historia. Instituciones. Documentos, 5 (1978), pág. 119; MARTín MARTín, J. L. y SÁNCHeZ EstÉveZ, J. M.: «Plasencia y su tierra en el siglo XV», Norba, 2 (1982), pág. 198.

18 A. Ch. Granada, leg. 577, $\mathrm{n}^{\circ} 24$, fol. 21v.

19 A. Ch. Granada, leg. 1371, no 5, fol. 12r. 
pondientes a unas veinticinco casas, quizás algunos menos. Hacia 1450, Martín Sancho había perdido claramente una parte de la población que había llegado a albergar, lo cual resulta sorprendente en una coyuntura demográfica alcista. Si pudo llegar a tener veinticinco vecinos, no parece que a mediados del XV superara los veinte. Aunque estamos hablando de magnitudes absolutas limitadas, porcentualmente el descenso es sensible.

Martín Sancho no sólo tiene una población reducida sino que presenta síntomas de sufrir un claro proceso de despoblación ${ }^{20}$. Gran parte de sus vecinos tienen una residencia de conveniencia, cuando no doble. La información se acumula sobre este aspecto. Conocemos el nombre de todos o casi todos los vecinos hacia 1450 y la procedencia de muchos de ellos. Veamos los datos siguientes ${ }^{21}$ :

VECINOS DE MARTIN SANCHO (c. 1450)

\begin{tabular}{|l|c|c|}
\hline VECINO & ORIGEN & $\begin{array}{c}\text { PERIODO DE } \\
\text { VECINDAD }\end{array}$ \\
\hline Alonso Martín Delgado & Miajadas & \\
\hline Andres González & & \\
\hline Aben Gariel & Medellín & \\
\hline Fernan González & ¿Medellín? & 2 años \\
\hline padre de Fray Negro & ¿Medellín? & \\
\hline Garcia de Merçagil & & \\
\hline Juan Barrero & & \\
\hline Juan Fernandez Cerrosón & & \\
\hline Juan García & & \\
\hline Juan Garcia el Calvo & & \\
\hline Juan García Varanco & & \\
\hline Juan Garcia Ventrinco & & \\
\hline Juan Gómez & & $3-4$ meses \\
\hline Juan Mateos & & \\
\hline Pero García boyero & & \\
\hline 2 vecinos & Villanueva & \\
\hline un judío & Medellín & \\
\hline
\end{tabular}

20 Una dinámica similar se documenta en Fuenteungrillo (VALDEÓN, J.: «Un despoblado castellano del siglo XIV: Fuenteungrillo», En la España Medieval, 3 [1982], pág. 713; REGLERO DE LA FuENTE, C.: «Los despoblados bajomedievales en los montes Torozos», págs. 195-6).

${ }_{21}$ Fundamentalmente hemos extraído los datos de A. Ch. Granada, leg. 577, no 24; información complementaria en DEL PINO GARCÍA, J. L.: Extremadura en las luchas políticas del siglo XV, Badajoz, 1991, doc. 2. Es posible que algún poblador aparezca duplicado, por los problemas que siempre plantea la identificación personal. 
Sobre algo menos de una veintena de vecinos cuantificados, un tercio procede de otros núcleos, especialmente de la tierra de Medellín y en particular de la villa. En ningún caso estamos ante una fuerte atracción poblacional, sino ante el interés en acceder a unos recursos abundantes debido al receso de la población. La vecindad en Martín Sancho supone una posibilidad magnífica para los propietarios de pequeñas cabañas vecinales. A veces se precisa este interés sin ambigüedad alguna. La que fuera mujer de Fernan González nos dice que «heran vezinos e estavan avezindados en el dicho logar de Martin Sancho por traer en los terminos del los ganados ${ }^{22}$. Un cristiano nuevo señala que oyó a su padre, que profesaba entonces la religión judía, "que si avia tomado vezindad en Martin Sancho en el tienpo que estava poblado que hera a cabsa de gozar del pasto del termino del dicho lugar» ${ }^{23}$. El padre de Fray Negro parece tener una doble residencia. No se indica que fuera vecino de la aldea sino simplemente que tenía una casa, yendo en fechas indicadas para aprovecharse del término y el pasto ${ }^{24}$. Del mismo modo, el hijo de Aben Gariel oyó decir a su padre que «avia tomado vezindad en el dicho logar a cabsa de poder gozar del termino de Martin Sancho e de los pastos del con sus ganados, aunque heran vezinos de Medellin» ${ }^{25}$. La población real de Martín Sancho parece, de este modo, que está sobrevaluada, pues una parte de la misma no residiría de modo continuo en el lugar y las razones de su residencia son, en todo caso, coyunturales.

A mediados del siglo XV, cuando el futuro Enrique IV cede la tierra de Medellín a Rodrigo Portocarrero, Martín Sancho es un núcleo que nunca ha superado un nivel modesto y que se encuentra en clara y franca regresión demográfica. Está al menos un $20 \%$ por debajo, quizás más, de su antiguo techo poblacional y una parte significativa de sus pobladores parecen tener una doble residencia. De este modo, aparecen todos los factores de riesgo de un proceso de despoblación. Ello explica la aparente facilidad con la que el nuevo señor de Medellín liquida la aldea.

La escasa población, que pudo llegar a los veinticinco vecinos o a algunos más, no impide que, a todos los efectos, Martín Sancho sea una aldea más de Medellín y que cuente con término y concejo propios. El término puede cartografiarse a grandes rasgos. De esta realidad deriva la existencia de una vecindad específica. Pese a los deseos de Medellín de considerar Martín Sancho como un mero barrio de la villa ${ }^{26}$, en conjunto no queda duda de que esto no era así.

22 A. Ch. Granada, leg. 577, no 24, fol. 55 r.

23 A. Ch. Granada, leg. 577, no 24, fol. 55r.

24 «oyo a su suegro Fray Negro que su padre del dicho su suegro tenia una casa en Martin Sancho en tienpo que estava poblado e que se yva algunas fiestas a holgar alla por gozar de los terminos e pastos del logar» (A. Ch. Granada, leg. 577, n 24, fol. 55r).

25 A. Ch. Granada, leg. 577, no 24, fol. 55r

26 Los oficiales de Medellín, según esta versión, «como en propios terminos de la dicha villa davan e dieron liçençias para hedificar casas para çerrar e haser viñas e huertas, e que syn liçençia de la dicha villa los vesinos del dicho logar de Martin Sancho ni otra persona alguna no osavan haser 
Un testigo señala que «vido dos honbres que heran de Villanueva se vinieron avezindar en Martin Sancho en tienpo que estaba poblado e el conçejo del lugar los resçibio e tuvieron la vezindad por tres o quatro meses, e que el conçejo de la villa no les dio liçençia ni ge la pidieron» ${ }^{27}$. Los vecinos de Medellín eran prendados si se introducían en sus comunales, como le sucedió a un vaquero y a otro vecino algunas veces ${ }^{28}$. Juan Fernández Cerrosón desempeñaba a mediados del siglo XV el cargo de montaraz ${ }^{29}$. Son bastantes los testigos que insisten en que Martín Sancho tenía sus términos «limitados e apartados de la villa de Medellin» ${ }^{30}$. La precisa delimitación del término y el avecindamiento de personas procedentes de la villa no puede entenderse de otro modo. Medellín y Martín Sancho tenían términos distintos y vecindades diferenciadas.

La estructura económica de Martín Sancho era similar en sus líneas generales a la del resto de las aldeas. Los diversos interrogatorios nos permiten conocer con cierto detalle el término y las diversas dedicaciones. Un testigo define en líneas generales la actividad económica al señalar que «labravan por pan e por vino, e criavan ganados ${ }^{31}$. En primer lugar debemos considerar los comunales. Junto a la aldea había «un exido pequeño alrededor del dicho logar»32 que constituiría el ejido ansarero. Es un espacio de limitadas dimensiones cuya finalidad es el mantenimiento de la cabaña vecinal y que no excluye necesariamente la existencia de otros ejidos. La aldea contaba con una dehesa boyal situada en la sierra Luenga o Alcornocosa, actual sierra de Troya, extendiéndose hasta cerca de la aldea. En verano se realizaba un coto o agostadero en la sierra «Azebuchosa», la actual sierra de Enfrente o de Remondo ${ }^{33}$.

casa ni çerrar huerta ni viña, sy lo tentavan de haser eran penados» (A. Ch. Granada, leg. 577, nº 24 , fols. 33r-33v), lo que es ratificado por los testigos presentados.

27 A. Ch. Granada, leg. 577, no 24, fol. 51v.

28 «no consentian a este testigo ni a otros pastores que entrasen a paçer dentro e sy entravan los prendan, e que lo sabe porque lo vido seyendo vaquero como dicho ha andando alrededor del termino e porque a este testigo mismo prendaron una ves o dos porque entro dentro a paçer con unas vacas, e oyo dezir este testigo asymesmo prendavan e prendaron a otros qualesquier que entrevan a paçer en el dicho termino» (A. Ch. Granada, leg. 577, $\mathrm{n}^{\circ} 24$, fols. 52v); «a este testigo mismo prendaron una ves o dos por que entro dentro a paçer con unas vacas, e oyo dezir este testigo asymesmo prendavan e prendaron a otros qualesquier que entrevan a paçer en el dicho termino» (A. Ch. Granada, leg. 577, no 24, fol. 52r).

29 A. Ch. Granada, leg. 577, no 24, fol. 51v.

30 A. Ch. Granada, leg. 577, no 24, fols. 50v-51r.

31 A. Ch. Granada, leg. 577, $n^{\circ} 24$, fol. 50v.

32 A. Ch. Granada, leg. 577, $\mathrm{n}^{\circ} 24$, fol. $52 \mathrm{v}$.

33 A. Ch. Granada, leg. 577, no 24, fol. 52r; «avia una sierra que se dezia la Syerra Luenga, e que hera dehesa boyal de los vezinos e moradores de Martin Sancho, la qual dicha Syerra Luenga hera desde desde la punta della que estava junto con el camino que yba a Merida hasta la otra punta que yba a dar en el pozuelo Salobre, e de la una parte de la falda de la dicha syerra las dehesas de Minga Gil e el Rincon e de la otra hasta dar çerca del sytio donde hera el dicho lugar de Martin Sancho a unas peñuelas que ay estan. Que en los veranos los dichos vezinos de Martin Sancho hazian cotos para sus bueyes en la Syerra Azauchosa» (A. Ch. Granada, leg. 1083, nº 7, fol. 9v). 
Junto a la aldea, en forma alargada siguiendo el arroyo de Cagánchez, aparecen unos espacios de cultivo intensivo «las çercas o çerquijos e viñas e otras cosas» que tendrían una extensión de "nueve o diez hanegas de trigo en senbradura» ${ }^{4}$. Se alude a la existencia de «doze viñas y huertas», en cuyo caso no todos los vecinos dispondrían de ellas ${ }^{35}$. Esta zona de espacios cercados se situaba próxima o colindante al ejido ansarero y tenía una extensión limitada.

La mayor parte del espacio agrario está constituido por las tierras cerealícolas. En ellas parece obtenerse sobre todo trigo y cebada ${ }^{36}$. Se extienden por la vega y, de forma dispersa, por todo el termino. La vega está limitada por el charco de Pedro Guisado, en el Guadiana, este río, el arroyo de Cagánchez, la quebrada del Guadiana (seguramente la actual quebrada de la Galapaguera), las sierras de la Cueva y la Acehuchosa, y el camino Arroyomolinos-Medellín (la actual carretera Medellín-Santa Amalia) ${ }^{37}$. Diversas propiedades aparecen en el límite norte de esta vega, junto a la quebrada del Guadiana, entre la llamada sierra Quesera, el arroyo de Cagánchez y los caminos de Arroyomolinos y Almoharín; una de ellas estaría junto a este arroyo. Seguramente estamos ante propiedades relativamente extensas, puesto que se definen por accidentes físicos o $\operatorname{caminos}^{38}$.

No esta claro si el espacio indicado constituía todo el terreno agrario de Martín Sancho. Al norte de la vega y de la sierra Aceuchosa se extendía hasta la cañada y la dehesa de la Patilla un extenso terreno cuya dedicación desconocemos. La vega es un espacio donde se había desarrollado la propiedad privada.

34 A. Ch. Granada, leg. 577, no 24, fol. 21v.

35 A. Ch. Granada, leg. 1371, n ${ }^{\circ}$ 5, fol. 12r.

36 «e conosçio labrarse las dichas tierra de la vega e coger trigo e çevada»; «un pedaço de tierra en ella que la poseya por suya un Pero Mexia, vesyno de Medellín, que la senbrava de çebada e sera un pedaço en que abria syete o ocho hanegadas de senbradura» (A. Ch. Granada, leg. 577, $\mathrm{n}^{\circ} 24$, fol. $45 \mathrm{r}$ ); «heredamientos e tierras de pan llevar de personas particulares que cogian çevada e trigo» (Id., fol. 46r).

37 «desde el charco de Pero Guisado andando de la ribera de Guadiana hasta dar en el arroyo de Cahanches, e por hese arroyo arriba hasta dar a la quebrada que dizen de Guadiana, e va por la quebrada arriba hasta la Syerra de la Cueva, e de alli buelve por la falda de la Syerra Azauchosa hasta el camino que va desta villa Arroyomolinos e por el dicho camino buelve al charco de Pero Guisado» (A. Ch. Granada, leg. 1371, n 5, fol. 11). En un sentido prácticamente idéntico, A. Ch. Granada, leg. 577, $\mathrm{n}^{\circ} 24$, fol. 46r.

38 «una tierra de pan llevar en la Syerra Azauchosa, desde los azauches hasta el llano; otro pedaço de tierra desde el camino que va Arroyomolinos desde la quebrada de Guadiana por ella arriba hasta el camino que va Almoharin, y por el dicho camino hasta en par de la syerra Quesera, e por alli atravesando derecho a la dicha Syerra hasta el dicho camino, e buelve por el hasta tornar a la dicha quebrada; otro pedaço desde la quebrada del Guadiana por el dicho camino de Arroyomolinos hasta dar a la dicha Syerra Quesera, e atravesando por ella hasta dar al dicho Arroyo de Cahanches, e por el dicho arroyo abaxo hasta donde buelve a juntar en la dicha quebrada; otro pedaço de tierra desde el arroyo de Cahanches en derecho de pozuelo Salobre por el dicho arroyo abaxo hasta unas peñas que estan junto con el dicho arroyo, çerca del camino que va a Merida, e de la otra parte el exido ansarero del dicho logar» (A. Ch. Granada, leg. 1371, nº 5, fols. 11v-12r) 
Sin embargo, en las zonas de baldíos o en terrenos no apropiados por particulares, los vecinos labraban tierras que seguramente dejaban tras la cosecha. Nada concreto sabemos sobre esto, pero un testigo además de señalar la vega como un espacio que formaba un pago agrario, nos dice sobre los vecinos «que labrava cada uno por do queria» ${ }^{39}$. El espacio cultivado sería, de este modo, mayor que el apropiado, que se circunscribía sobre todo a la vega y a espacios próximos a las quebrada del Guadiana y al arroyo de Cagánchez.

Junto a la actividad agraria, la dedicación ganadera era fundamental. Por un lado, el descenso de población de Martín Sancho ha debido suponer un aumento de los pastizales. No debemos olvidar la razón de algunos avecindamientos. La ganadería ha sido, sin duda, un sector de importancia creciente conforme la población ha ido disminuyendo y con ello el espacio cultivado. Alonso Martín Delgado, originario de Miajadas, puede servirnos de ejemplo. Su hijo se encargaba de cuidar sus «puercos e cochinos» ${ }^{40}$. Al este y norte del término parecen abundar los terrenos de uso ganadero. La actividad agraria parece concentrarse en la zona suroeste.

La comunidad de Martín Sancho presenta un perfil diversificado. La oligarquía medellinense, o simples vecinos, disfrutaban de importantes propiedades. Algunos vecinos de la aldea también disponían de una cantidad elevada de tierras cultivables, mientras otros eran simples asalariados rurales. Dentro del primer grupo, Pedro Mejía contaba con una parcela de siete-ocho fanegas que hacía sembrar por sus «operadores» ${ }^{41}$. Este personaje era uno de los miembros más cualificados de la oligarquía. Nieto de Gómez González Rengel, hijo de Diego González Mejía y María Sánchez de Sandoval, y padre de Diego Mejía, señor de Don Llorente, y Juan Núñez de Prado, contaba con importante propiedades entre las que se incluían varias dehesas ${ }^{42}$. Don Juan Pacheco, tras recibir la villa de Medellín, le nombró alcaide de la fortaleza en enero de $1446^{43}$; este mismo año aparece como procurador de la villa ${ }^{44}$. Estamos, por tanto, ante una persona de gran riqueza y poder. Hernando González de Morera, vecino también de Medellín y sobre el que se señala «que tenia muchas tierras en la dicha vega» ${ }^{45}$, aunque no disfrutara de una situación tan preeminente debía

39 «no tenian ninguna tierra cosnoçida salvo que labrava cada uno por do queria e que en toda la dicha vega no quedava ninguna cosa por labrar»(A. Ch. Granada, leg. 1371, n 5, fol. 45r). Esto quizás explique que Medellín afirme, en contra de la sentencia del 14 de febrero de 1520, que los vecinos de Martín Sancho «no tenian tierras de pan levar proprias, salvo que tubiera conçejil, e alçado los panes quedavan para el conçejo, el qual lo repartira cada año entre los vezinos» (Id., fol. 32r).

40 A. Ch. Granada, leg. 577, $\mathrm{n}^{\circ} 24$, fol. $47 \mathrm{v}$.

41 A. Ch. Granada, leg. 577, no 24, fol. 45 r.

42 Sobre Pedro Mejía y su familia, cf. Clemente Ramos, J.: «Valdetorres, de dehesa a aldea (1409-1510)» (lamentablemente, no se ha incluido en la publicación por error de edición un cuadro genealógico de la familia Mejía).

43 Del Pino García, J. L.: Extremadura en las luchas políticas..., doc. 2, págs. 327-8.

44 Del Pino García, J. L.: Extremadura en las luchas políticas..., doc. 2, pág. 314.

45 A. Ch. Granada, leg. $577, n^{\circ} 24$, fol. 45 r. 
pertenecer igualmente a los sectores más beneficiados de la villa. En 1446 aparece como regidor ${ }^{46}$. También tenía propiedades en Martín Sancho, el arcipreste de Medellín ${ }^{47}$.

El resto de los propietarios eran vecinos del lugar. Parece haber unanimidad en que la persona más rica era Juan García el Calvo ${ }^{48}$. Posiblemente, esta circunstancia era la que le permitía ser alcalde con frecuencia. Según un testigo, habitualmente se alternaba en el cargo con Juan Barrero. Todo nos hace pensar que este último también tendría una buena base económica. También se citan como propietarios Juan Garcia Ventrinco y Garcia de Merçagil ${ }^{49}$. Llama la atención el pequeño número de vecinos citados como propietarios. Hay un sector de reciente inmigración que parece estar interesado sobre todo en las posibilidades ganaderas del término. Este grupo a su vez quizás tuviese propiedades en sus lugares de origen. En algún caso se precisa la condición de asalariado $^{50}$, lo que necesariamente no implicaría a toda la familia.

La escasa entidad demográfica de Martín Sancho no impide que estemos ante una comunidad socialmente diversificada. Frente a las propiedades poseídas por miembros de la oligarquía de la villa, aparecen algunos vecinos que seguramente deberíamos situar en el nivel de los campesinos enriquecidos o acomodados. Sin embargo, el acceso a la propiedad agraria no parece generalizada. El grupo que dispone de escasas propiedades, o de ninguna, estaría formado tanto por personas empobrecidas como por otras más interesadas en las posibilidades ganaderas del término.

Martín Sancho, como la mayor parte de las aldeas de la tierra de Medellín, contaba a mediados del siglo XV, inmediatamente ante de despoblarse, con un único alcalde ${ }^{51}$. No parece que hubiera otra entramado institucional salvo la propia asamblea concejil. En 1446, cuando Medellín y sus aldeas prestan reconocimiento a su nuevo señor Juan Pacheco, marqués de Villena, por Martín Sancho juran «Juan Gomes, alcalde de la dicha aldea» y dos vecinos «por si e en nombre de los vesinos de la dicha aldea de Martin Sancho»52. El mismo esquema se repite para el resto de las aldeas, que tampoco cuentan con regidores ni procurador. La alcaldía del lugar parece estar monopolizada por una muy

46 Del Pino García, J. L.: Extremadura en las luchas políticas..., doc. 2, pág. 314.

47 A. Ch. Granada, leg. 577, no 24, fol. 45r.

48 A. Ch. Granada, leg. 577, n ${ }^{\circ} 24$, fol. 51v: «conosçio a uno dellos que hera el mas rico e se llamava el Calvo».

49 A. Ch. Granada, leg. 577, no 24, fol. 45 r.

50 «seyendo este testigo mançebo, estando a soldada en el logar de Martin Sancho, que a la sazon estava poblado» (A. Ch. Granada, leg. 577, no 24, fol. 50v).

51 DEL Pino García, J. L.: Extremadura en las luchas políticas..., doc. 2, pág. 332.

52 DEL Pino García, J. L.: Extremadura en las luchas políticas..., doc. 2, pág. 331. La alusión a los «alcaldes e regidores» de Martín Sancho en un interrogatorio presentado por María de Sotomayor, segunda mujer de Alonso Fernández Portocarrero, y sus hijos es seguramente un anacronismo que remite a la estructura concejil aldeana existente a principios del siglo XVI (A. Ch. Granada, leg. $577, \mathrm{n}^{\circ} 24$, fol. $\left.51 \mathrm{r}\right)$. 
modesta oligarquía aldeana. Dos vecinos parecen turnarse en ella, Juan García el Calvo y Juan Barrero ${ }^{3}$, lo que no impediría el acceso de algún otro. Al menos en el primer caso, y quizás también en el segundo, debemos suponer que la riqueza fue un elemento central en este protagonismo. En todo caso, no podemos dar a este detalle un significado excesivo. El número de vecinos en condiciones de desempeñar el cargo debió ser muy limitado. Una parte significativa llevaba residiendo en el lugar poco tiempo y su interés exclusivo se dirigía hacia el aprovechamiento ganadero. Por otro lado, las atribuciones de los alcaldes aldeanos eran limitadas. Sólo juzgaban pleitos civiles de cuantía reducida ${ }^{54}$. El poder del concejo aldeano y, en particular, del alcalde a mediados del siglo XV era escaso. Con todo, la élite aldeana disfrutaba de una claro protagonismo.

En síntesis, podemos señalar que Martín Sancho, que nunca dejó de ser un núcleo de nivel moderado, presentaba a mediados del siglo XV evidentes signos de crisis. La población estaba claramente por debajo de lo que debio ser su nivel más elevado. Si excluimos a los vecinos de conveniencia, que quizás sólo residiesen de forma discontinua, se detecta un claro receso demográfico. Esta circunstancia será fundamental para entender la actuación de Rodrigo Portocarrero.

\section{ViOLENCIA SEÑORIAL Y DESPOBLACIÓN: LA DEHESA DE MARTÍN SANCHO (1449- 1517)}

En agosto de 1449, la tierra de Medellín entra en una nueva etapa. El príncipe y futuro rey Enrique nombra gobernador de la misma a Rodrigo Portocarrero y se la dona meses después ${ }^{55}$. Hasta entonces, Medellín ha estado durante largos periodos bajo la dependencia señorial de altos personajes descendientes de la realeza, pero ahora el beneficiado es un personaje oscuro que conseguirá acceder a la alta nobleza.

El nuevo señor jurisdiccional va a proceder a despoblar Martín Sancho. Algunos testigos indican que la actuación se realiza siendo aún gobernador, por tanto, antes de recibir la autoridad jurisdiccional en noviembre de $1449^{56}$. La

53 «avia dos vezinos el uno que se llamava Juan Barrero e el otro el Calvo... que heran alcaldes del logar e uno hera el un año e otro el otro año» (A. Ch. Granada, leg. 577, n 24, fol. 51r).

54 «oya a los vezinos del logar que livravan algunos pleytos hasta en çierta contia... que les vido traer varias contias e los vido librar e oyr algunos pleytos çeviles de poca contia entre los vezinos del logar» (A. Ch. Granada, leg. 577, no 24, fol. 51r).

55 Arch. Ducal Medinaceli, Medellín, legs. 6, $\mathrm{n}^{\circ} 15, \mathrm{y} 1, \mathrm{n}^{\circ} 3$.

56 «quando el dicho logar de Martin Sancho se despoblo el señor rey Don Enrique e el don Rodrigo Portocarrero, como su governador e justiçia mayor en el dicho nombre, como cosa bacante e despoblada tomo posesion para el dicho señor rey del dicho lugar, terminos e dehesas e exido, tierras, casas, viñas, heredades particulares e de todo ello hizo dehesa heredamiento para el dicho señor rey e para su patrimonio real» (A. Ch. Granada, leg. 577, $\mathrm{n}^{\circ} 24$, fol. 50r); un testigo señala que el gobernador era Pedro Portocarrero, hermano de Rodrigo Portocarrero, lo que puede tratarse de un error o de la asignación de responsabilidades dentro del entorno familiar (Id., fol. 50v). 
violencia señorial ha jugado un papel fundamental. La versión de Medellín es clara y unívoca, vinculando despoblación, violencia y apropiación señorial: «la fuerça hecha por el dicho conde que avia despoblado al dicho lugar de Martin Sancho, e que con codiçia e avariçia avia quitado a los vezinos su bibienda y heredades, e que avia derribado las casas del dicho lugar, e hecho llevar la teja e madera a su fortaleza, a avia vendido los panes juntamente con las yerbas a pastores e serranos, a avia talado las viñas e las huertas e fecho otras muchas tiranias» ${ }^{57}$. Un testigo aludirá igualmente a la violencia ejercida sobre los vecinos, de los que ha recibido la información ${ }^{58}$. Si bien, no parece haber dudas sobre la actuación del gobernador, pronto señor y futuro conde, y sobre la presión ejercida sobre los pobladores de Martín Sancho, la violencia no fue el único mecanismo utilizado. Parece que las heredades individuales fueron adquiridas aunque bajo las circunstancias señaladas. Es llamativo que en una sentencia de 1520, en el pleito que mantienen Alonso de Monroy (descendiente de Alonso Fernández Portocarrero, hermano de Rodrigo Portocarrero) y Medellín, la parte ganadora, se concede al primero «veynte hanegadas de tierras que sean junto al arroyo de Caguanches, las quales le den y entreguen por razon de los huertos, e viñas, e casas, y otras qualesquier tierras y heredades que tenian qualesquier personas particulares en el lugar de Martin Sancho" 59 . Se diferencia nítidamente entre el término, que recibe Medellín, y el derecho de los descendientes de Alonso Fernández Portocarrero sobre los heredamientos privados. Todos nos hace pensar que Rodrigo Portocarrero compró estos heredamientos, aunque posiblemente a un precio limitado por la fuerte presión ejercida. Con o sin compras, la violencia es el elemento primordial que explica la definitiva y rápida despoblación de Martín Sancho.

La finalidad de la actuación de Rodrigo Portocarrero es clara: la apropiación de un término como propiedad particular y su conversión en dehesa. El príncipe Enrique al ceder la tierra de Medellín no ha debido transmitir ninguna, o casi ninguna, propiedad solariega. Los sucesivos señores jurisdiccionales de Medellín, incluida la monarquía, se han ido desprendiendo de gran parte de sus propiedades si no de todas. Por ello, Rodrigo Portocarrero sólo recibirá rentas. Una de las líneas fallidas de la futura familia condal es proveerse de una sólida base solariega ${ }^{60}$.

Una vez despoblado Martín Sancho, Rodrigo Portocarrero se apropió el término y lo convirtió en dehesa ${ }^{61}$. Cuando poco después lo cede a su hermano,

57 A. Ch. Granada, leg. 1083, fol. 12r.

58 «que despues que el conde despoblo Martin Sancho vido quexarse a los vesinos del disiendo que el conde los avia mucho agraviado en los echar de sus casas e despoblar a Martin Sancho» (A. Ch. Granada, leg. 577, $n^{\circ} 24$, fol. 48r)

59 A. Ch. Granada, leg. 1371, fol. 30r.

60 Beatriz Pacheco se apropió, siendo viuda de Rodrigo Portocarrero, de la dehesa de Don Salvador y transitoriamente de Valdetorres, perteneciente a los descendientes de Gomez González Rengel (Cf. Clemente Ramos, J.: «Valdetorres, de dehesa a aldea [1409-1510]», págs. 60-1).

61 La conversión de los despoblados en dehesa, algo generalizado en Medellín en consonancia con su modelo socioeconómico, también se documenta en Ávila (LORENZO PINAR, F. J. y IZQUIERDo MI- 
Alonso Fernández Portocarrero, aparece «con todas sus entradas e salidas, usos e costunbres, pastos e prados, aguas manantes e corrientes, e estantes» ${ }^{62}$. En unos años, nada queda en pie de lo que fue el hábitat aldeano y sus explotaciones agrarias ${ }^{63}$. La nueva dehesa se arrendará, parece que sólo parcialmente, a ganaderos mesteños o riberiegos. El conde también lleva allí su ganado ${ }^{64}$.

Como hemos indicado, Martín Sancho permanece en poder de Rodrigo Portocarrero poco tiempo. En 1451, acuerda con Juan de Sotomayor, señor de Alconchel, el matrimonio de su hermano Alfonso Fernández Portocarrero con María de Sotomayor, hija de Alfonso de Monroy y Juana de Sotomayor, y se compromete a cederle esta propiedad, cuyo rendimiento se calcula en treinta mil maravedís anuales ${ }^{65}$. La cesión se hace efectiva en $1454^{66}$. El control de Martín Sancho por Alonso Fernández Portocarrero y sus descendientes a lo largo de la segunda mitad del siglo XV será compatible con un cierto aprovechamiento por parte de Medellín, al menos hasta la última década del siglo $\mathrm{XV}^{67}$. Quizás esto tenga que ver sólo con la bellota, sobre la cual no tienen los propietarios de dehesas ningún derecho preferente.

En torno a la fecha en que los descendientes de Alonso Fernández Portocarrero imponen en Martín Sancho su aprovechamiento exclusivo, empieza a gestarse la lucha de Medellín por su posesión. Se perfila una disputa entre dos derechos excluyentes: la apropiación de un término despoblado por la villa y tierra o por la autoridad jurisdiccional ${ }^{68}$. Este conflicto se materializa en un contexto particular. En la última década del siglo XV se va a desarrollar un enfrentamiento abierto entre una sector posiblemente mayoritario de la oligarquía medellinense, liderada por Juan Núñez de Prado, y del común de la villa y

SIEGO, J. I.: «Términos redondos y despoblamiento en Ávila», pág. 269), pero dista de ser un hecho generalizado en la corona de Castilla, donde frecuentemente estos términos siguen cultivándose.

62 Arch. Chancill. Granada, legajo 2072, n ${ }^{\circ}$ 6, fol. 1 r.

63 Un testigo señala que tras la despoblación «nunca mas vio labrarse la dicha vega» (A. Ch. Granada, leg. 577, no 24, fol. 45v).

64 «paçia el dicho termino con sus vacas» («(A. Ch. Granada, leg. 577, $\mathrm{n}^{\circ} 24$, fol. 37v).

65 A. Chancill. Granada, legajo 2072, $\mathrm{n}^{\circ}$ 6, fols. 5r-6r.

66 A. Chancill. Granada, legajo 2072, $\mathrm{n}^{\circ}$ 6, fol. 1.

67 «este testigo syendo boyero de Medellin comio de Remondo en el dicho termino con los bueyes e vacas de el, e asymismo vido comer la vellota con los puercos de Medellin, e aun vido mas que otros boyeros de Medellin que fueron antes que el yvan con la boyada al termino de Martin Sancho e ponian el hato a mojon de Patilla e de Remondo e de Martin Sancho, e de alli comian el termino de Martin Sancho con los buees e vacas de Medellin hasta que despues Alonso Hernandes Puertocarrero, puede aver dose o treze años, ha vedado todo lo susodicho a vezinos de Medellín» (A. Ch. Granada, leg. 577, no 24, fol. 39v).

68 Diversos testigos ratifican la pregunta de Medellín en la que se señala «que todos los terminos del tal logar que ay quedan yermo e despoblado se aplican a los terminos del lugar mas cercano del dicho logar despoblado e que es de su jurediçion» (A. Ch. Granada, leg. 577, no 24, fol. 44r); la parte propietaria sostiene, sin embargo, una posición contraria (A. Ch. Granada, leg. 577, $\mathrm{n}^{\circ} 24$, fol. 50r. -cf. nota 56-) 
tierra, por un lado, y el conde Juan Portocarrero, por otro. Esta alianza interclasista va a aprovechar la consolidación del poder monárquico que sigue a la victoria de los Reyes Católicos. El conflicto se vehicula fundamentalmente en el ámbito procesal, dentro de las Chancillerías y el Consejo Real. En este contexto, general y particular, comienza la reivindicación del término de Martín Sancho por el concejo de Medellín. En 1494, los monarcas encargarán al corregidor de Medellín que investigue ciertas apropiaciones de terminos por parte de «cavalleros e otras personas», incluyéndose Martín Sancho ${ }^{69}$. En 1504, una ejecutoria real reconoce los derechos de Medellín excepto sobre los heredamientos privados, sobre los cuales se absuelve a María de Sotomayor, mujer de Alonso Fernández Portocarrero, ya fenecido, y sus hijos Rodrigo Portocarrero, Alonso de Monroy y doña Elvira. La villa procederá a realizar la toma de posesión ${ }^{70}$.

Esta ejecutoria y posterior toma de posesión no ha supuesto la finalización del pleito. Lo continuarán los hijos de María de Sotomayor. En primera instancia el pleito se desarrolló ante la justicia ordinaria de Medellín. En un fecha indeterminada, quizás poco antes de 1517, Juan Guisado, alcalde ordinario, emite una sentencia favorable a Alonso de Monroy y sus consortes. Medellín la recurre. La Chancillería de Granada falla en 1517 «que sea tornada e restituyda a la dicha villa de Medellin la posysyon del heredamiento de Martin Sancho», lo que se realiza ese mismo año ${ }^{71}$. El pleito continúa en relación con los heredamientos privados, sobre lo cual Alonso de Monroy (considera insuficiente lo obtenido) y Medellín (estima que no los había) mantienen posiciones encontradas. En todo caso, el control del término de Martín Sancho por Medellín se convierte ahora en definitivo, pasando a ser un ejido de la villa ${ }^{72}$.

\section{CONCLUSIÓN}

La definitiva configuración del poblamiento medieval en Extremadura es un proceso complejo en el que bastantes aldeas terminan siendo abandonadas de modo natural. Muchos de ellas sólo aparecen en los momentos finales de su existencia o cuando se han convertido en despoblados. La mayor parte de la información disponible sobre Martín Sancho, aunque se documenta en la segunda mitad del siglo XIV, se obtiene más de cincuenta años después de su abandono a mediados del siglo XV.

La despoblación de Martín Sancho se produce a partir de una tendencia a largo plazo y una actuación señorial violenta. Sin ambos elementos no puede entenderse. Al igual que ocurre con otras aldeas de la tierra de Medellín que

69 A. G. Simancas, R. G. Sello, 1494-setiembre-20, fol. 257.

70 A. Ch. Granada, leg. 1083, no 7, fol. 34v.

71 A. Ch. Granada, leg. $1371, \mathrm{n}^{\circ} 5$, fols. $8 \mathrm{r}$ y $13 \mathrm{v}$.

72 «Martin Sanchez que es exido desta villa» (A. G. Simancas, Consejo Real, Leg. 140, exp. 2, fol. 130r). 
terminaron por despoblarse, Martín Sancho es una población de escasa entidad. Nunca debió superar un nivel demográfico modesto, lo que suponía un riesgo evidente para su permanencia. En la primera mitad del siglo XV es un núcleo en crisis. Su población presenta una disminución importante en términos relativos. Además, una parte de su limitada población posiblemente no estuviera asentada de modo efectivo o unico en la aldea, sino que tendría una vecindad de conveniencia y/o doble para aprovechar el término. Esta situación ha debido favorecer la acumulación de propiedades por la oligarquía medellinense. En todo caso, hacia mediados del siglo XV, Martín Sancho parece presentar una tendencia clara a la despoblación.

Esta coyuntura fue lo que hizo tan efectiva la actuación del gobernador y muy pronto señor de Medellín Rodrigo Portocarrero. La resistencia de la comunidad aldeana debió ser escasa. Una parte importante de los vecinos sólo residían en Martín Sancho, y quizás no de forma continua, desde hacía muy poco tiempo. Una actuación violenta decidida y la probable compra de los heredamientos privados debió ablandar su voluntad. A consecuencia de ello, el lugar se despuebla en una fecha precisa, entre agosto y noviembre de 1449. En todo el proceso, nunca se alude a los fenómenos epidémicos, aspecto sobre el cual las fuentes extremeñas guardan un silencio profundo sólo roto muy excepcionalmente. De todos modos, la despoblación de Martín Sancho se produce en una coyuntura general de crecimiento demográfico, de la cual es un ejemplo próximo Valdetorres.

¿Qué causas pueden explicar la dinámica de descenso demográfico que parece documentarse en Martín Sancho? Sólo podemos intuirlas. Las razones físicas (morfología, suelos) no parecen decisivas. Quizás la proximidad a Medellín haya sido un factor negativo. Aunque esta circunstancia no ha impedido la consolidación de Mengabril, las mayores aldeas de la tierra (Miajadas, Don Benito y Guareña) tienen una ubicación periférica respecto de la villa.

En síntesis, la evolución de Martín Sancho, salvo en su momento final, ha debido ser la de otros muchos lugares que terminan desapareciendo dentro del proceso de tanteo que ha ido definiendo la red regional de poblamiento. La proximidad a Medellín debió dificultar su consolidación. Su debilidad demográfica, creciente, facilitó que la violencia señorial fuera decisiva. 


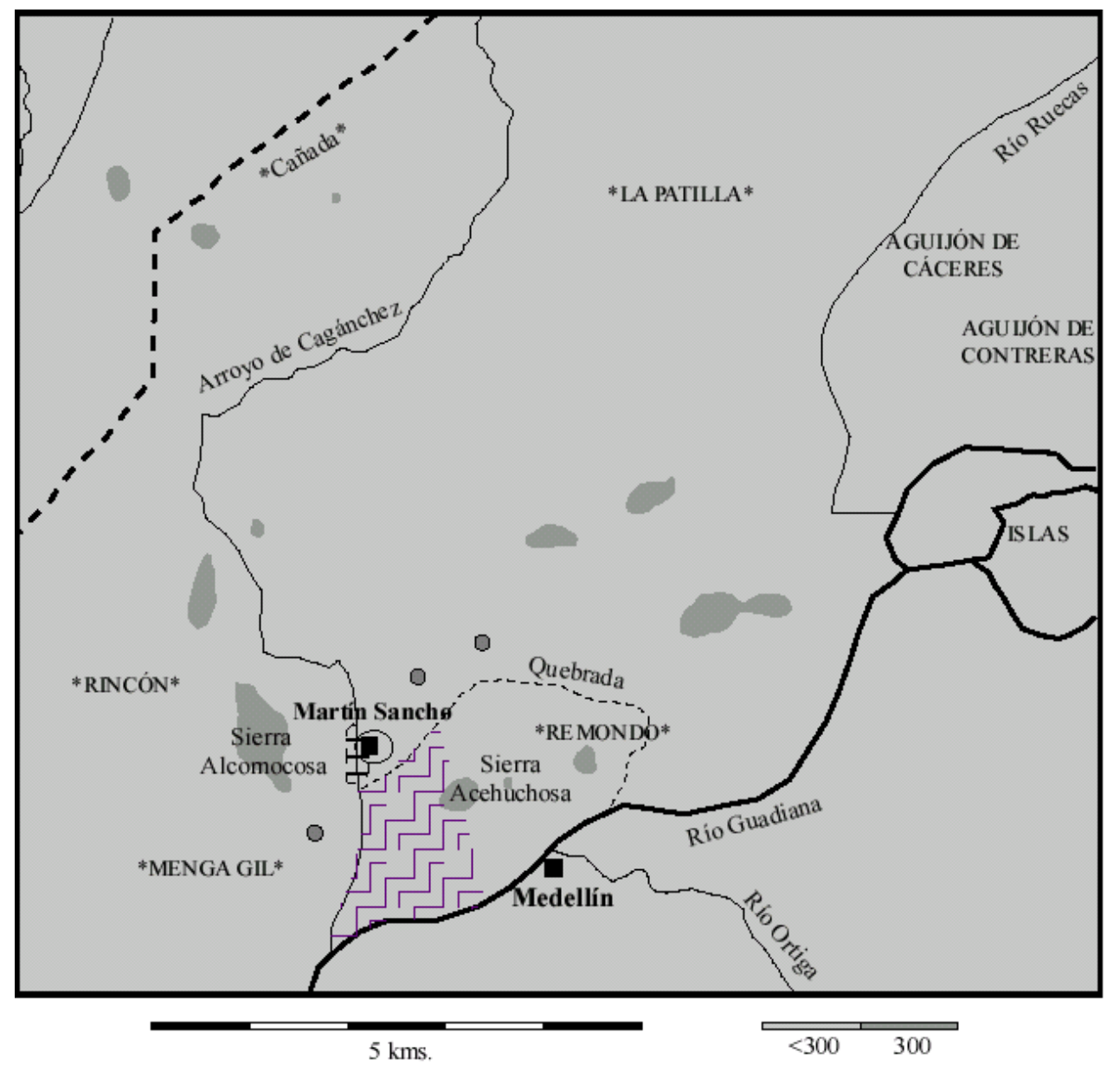

\begin{tabular}{|llcl|}
\hline \multicolumn{4}{|c|}{ Término de Martín Sancho (c. 1450) } \\
& Población & $\bigcirc$ & Ejido \\
Cercas & Propiedad particular & $* \ldots *$ & Lega \\
MENGA GIL & Dehesa & $-\mathbf{- ~ - ~}^{*}$ & Cañada \\
& & & \\
\hline
\end{tabular}

